\title{
从数字化到信息化的测绘学科进展
}

王琅

顺昌县地产开发公司

DOI:10.32629/gmsm.v2i6.387

[摘 要] 近年来,随着社会经济的快速发展,信息技术的进步,测绘学科已经从以前的模拟测绘转变为了现在的数字化测绘,并逐渐向信息化测 绘方向发展和转变。

[关键词] 测绘学; 信息化; 测绘数字化; 测绘进展研究

\section{1 信息化测绘技术的重要性}

测绘行业一直以来都是社会各界活动中一项基础服务型的行业, 其先 进性主要表现在我国科学研究、经济建设以及国防建设等各个应用领域, 据相关资料显示, 人类生活中各项活动的信息获取都离不开其自身空间位 置的明确, 假设把相关信息依附在这些空间数据基础设施中具体的逻辑平 台中, 能够实现不同空间位置信息的发掘和交流。基础地理信息作为空间 数据基础设施中的具体核心内容, 因其具有广泛的基础创造性, 现已经发 展成为了能够适应社会发展应用战略的需求和信息社会中活力发展点的 内容之一。提出信息化测绘这一概念, 是针对我国当前现代化测绘的社会 需求以及发展形式所提出的, 所谓信息化测绘主要是指在数字化测绘的建 筑的基础上所构建的, 在完全网络化运行的条件下, 有效、实时地为社会各 类用户提供相应的地理空间信息综合服务的一种测绘方式, 信息化测绘方 式不仅涵盖了数字化测绘技术的所有特点, 同时还具有一些数字化测绘 技术所不具备的特征 ${ }^{[1]}$ 。信息化测绘体系将获取的地理空间信息以及数 据库的建设, 从静态生产转化为实时更新与动态变化监测, 由原来的基 础设施升级和改造成为了一种适应当前社会功能公共使用的设施, 从数 字化测绘技术发展到信息化测绘技术的这一过程, 是一项重大科学技术 的变革历程。

\section{2 数字化测绘与信息化测绘二者的差异性}

2. 1 信息采集技术的差异性

在实际的工作中, 数字化测绘从结果上来看更加重视的是数字化的特 征, 在对信息进行采集的时候, 可以使用以往的信息数据,也可以使用先进 设备处理之后的数据, 所以数字化测绘在实际的工作中对信息采集的相关 技术要求并不是非常的严格, 一般情况下只要输出的结果能够以数字的形 式进行显示, 就可以实现其功能。信息化测绘和数字化测绘实际上在采集
技术方面还是有着非常显著的不同, 信息化测绘使用的是最为先进的技术, 这样就可以使得测绘的准确性大幅度提升, 从而也提高了测绘的质量和 水平 ${ }^{[2]}$ 。

\section{2产品的差异性}

数字化测绘主要的依托是自动化测绘技术, 在使用局域网的过程中对 信息进行搜集和整理, 所以范围并不是很大。而信息化也是依靠自动化技 术, 但是全网络范围使得其产品涉及的领域更加的宽泛, 同时, 也提供了更 多的资源, 对资源的整合工作而言也有着十分重要的意义。数字化测绘产 品当中更多的是单机处理, 这样就会使得有很明显的局限但是信息化测绘 比较重视的是整个网络资源的汇总和整理, 这样也使得资源的利用率得到 了显著的提升 ${ }^{[3]}$ 。

\section{3 数字化到信息化的测绘学科发展进程以及确定目标}

3.1 发展进程

总的来说, 数字化测绘体系可以把空间各类数据信息整合在一起, 并 把3S技术和资源当做其实际的重心内容, 广泛的使用该网络存储技术, 全 方位的收集并整理更为详细的数据信息, 对这些数据信息进行加工, 让其 应用管理可以朝向数字化的发展进程。其相关产品的获取形式也和传统的 纸质地图有着很大的差别, 其是一种新型的现代 $4 \mathrm{D}$ 产品。信息化测绘技术 的应用可以有效的推动我国数字化的测绘技术的发展, 延续其技术的发展 优势。无论是在具体技术方面, 还是在其实际的生产流程方式方面进行分 析, 信息化测绘技术体系都能符合其现阶段科学技术的发展规律。目前, 信息化测绘技术主要是通过多种渠道来实时的收集整理多源空间数据信 息, 将这类数据信息支撑起系统。其技术方式为大规模自动智能化信息管 理的重要方式, 可以构建出一个较为完整的, 且内容十分丰富的地理空间 信息产品模型。信息化测绘技术的使用会提供给人们更为便捷性的服务,

飞艇遥感测绘, 有效的融合了无人机技术与遥感技术, 是一种全新的 迅速取得低空较高分辨率遥感数据信息的方式。此技术具备专用化、低费 用、小型化、迅速以及即时监测等相关优势, 可作为卫星遥感和常规航空 遥感的重要补充手段。为了全面贯彻国家测绘地理信息总局 “监测地理国 情、创建数字中国, 壮大产业、创建测绘强国” 的整体目标, 国家测绘技术 中心和中航工业特种飞行器研究所签署了合作协议, 成立了相应的工作 小组。依托中航工业特种飞行器研究所具备的技术优势, 促进飞艇遥感 信息系统的产业化发展, 为国家测绘地理信息局提供全新的设备、全新 的技术保障, 也为我们国家的地理国情监测以及应急保障工作奠定较好 的装备基础。

\section{3 结语}

开展地理国情监测, 是历史赋予测绘工作的别无选择的新使命。利用 现代测绘技术, 能够准确地反映各类资源、环境、生态、经济要素的空间

分布及其发展变化规律, 为我国的地理国情监测提供具有参考价值的数 据。只有以国家现代化测绘基准体系为基础, 才可以为地理国情监测提供 丰富的测绘基准信息, 最终能够满足经济社会对基准信息的具体要求。由 此可见, 测绘技术贯穿于地理国情监测的整个过程, 综合应用各项技术, 能 够保证监测成果的客观、准确、可靠, 更好地为社会公众服务, 并且得以促 进地理国情监测工作的顺利进行。

\section{[参考文献]}

[1]黄好,杨泉.分析地理国情监测以及测绘技术实践 [J]. 低碳世 界,2014(23):175-176.

[2]王亚雄. 探析地理国情监测以及测绘技术实践 [J].城市建设理论研 究(电子版),2017(18):132.

[3]张俊宇,梁寿强, 周亿萍. 遥感与GIS技术在地理国情监测中的应用与 研究[J].低碳世界,2017(03):85-86. 
实时的开展网络安全设置工作, 使得人们的日常生活变得更加的多元化, 其实际的信息服务也更具人性化的特征。

3. 2 确定目标

构建信息化测绘技术系统目的主要就是为了人们可以随时随地的分 享自己所需要的各类地理服务数据信息内容。首先, 测绘技术学科在实际 的发展时, 应当构建出一个综合性较强的, 且多层次地理观测的平台, 其平 台的观测内容比较复杂, 所涉及到的层面也比较广泛, 其平台还具有较高 的影像截获性能。另外, 其还应当妥善合理的处理各类数据信息, 组建完整 的技术系统, 通过多源观测数据处理系统的使用来更为精确的分析以及处 理各类信息测绘技术, 以此来更好的提升其技术的发展水平。尽可能的突 破关键技术, 共享地理空间数据信息, 开发使用一些带有自主知识产权的 信息化测绘软件,让其地理信息的服务能力得到增强, 丰富其原本的地理 信息内容。另外, 相关的工作人员还应当深入的探究网络地理空间信息的 管理技术以及数据模型基础理论等, 实时的传递多维时空的信息, 设定好 空间信息集成的标准规范, 打造信息集成硬件具体平台。

\section{4 数字化测绘基础上信息化测绘的服务特性}

现阶段, 我国所使用的测绘技术的服务保障性能比较差, 其需要在一 定程度上提升该项能力。在实际所开展的信息化测绘体系中, 要对相应的 战略任务进行科学高效的规划和设计,让其技术都在各个工程建设领域中 发挥出自身最大的效用, 确定好测绘行业发展的首要目标。在数字化测绘 基础上信息化测绘的服务特征为以下几点: 第一, 提升服务保障层级, 信息 化测绘技术能够使测绘服务系统相对应的层次有效地体征, 在明确测绘技 术系统辅助地位的时候, 利用测绘的支撑作用来强化和推动我国测绘事业 的发展。第二, 变化服务保障模式, 测绘技术体系模式的改变主要有后续服 务模式的转变、空间信息应用模式的转变等。第三, 提升了服务保障质量, 其主要表现在产品服务的优化以及不断增值, 使得相关空间数据信息的信 息内容和质量能够满足当前社会发展的需求, 特别是对空间信息现势性的 改变尤为明显。此外, 还加强了其服务保障的效果, 确保了各保障服务的有 效、及时和合理。

\section{5 数字化测绘学科到信息化测绘学科的研究发展}

5.1 建立现代化的测绘体系

建立现代化的测绘体系, 才能实现数字化测绘到信息化测绘的转变。 现代化的测绘体系作为测绘学科的基础, 在地理信息获取方面, 提高了空
间位置、重力、高程等众多环节信息数据的可靠性。空间位置、重力、高 程等作为地理信息判断的主要根据, 其可靠性对于空间地理信息判断准确 性有着重要影响 ${ }^{[4]}$ 。

5.2 摄影测量与遥感测量技术的研究发展

目前, 数字化测绘与信息化测绘中使用的主要图像收集方法为摄影测 量技术以及遥感测量技术, 通过摄影测量技术或遥感测量技术得出的图像 数据, 可以支持数字化测绘与信息化测绘输入, 通过数字化测绘与信息化 测绘的处理、加工输出地理空间模型产品。

综上所述, 信息化测绘作为国防建设、社会发展以及经济建设的基础 事业。在未来的发展中有着特别广泛地前景, 但同时也面临着非常严峻的 挑战。这就要求在数字化测绘向信息化测绘的发展进程中, 我们必须要克 服各种不利的因素, 有效地调动各方面积极因素, 促进地理信息产业和测 绘事业的发展, 真正意义上实现信息化测绘。

\section{6 结束语}

数字化技术为人们提供了良好的基础, 在信息化测绘全新发展阶段, 离不开数字化模式, 只有全面融合二者优势, 发挥各自长处, 才能在完全网 络化运行环境中向社会与用户提供更加全面、准确的信息服务产品, 特别 是在地理空间信息、功能信息等数据建设中, 信息化技术更加优良, 可以说, 信息化测绘全面推动了数字化测绘进步,有效实现了我国测绘学科跨越式 发展, 满足全社会需求。

\section{[参考文献]}

[1]赵鹏.测绘新技术在建筑工程测量中的运用思路[J].城市建设理论 研究(电子版),2018,(01):105.

[2]陈大鹏.测绘新技术在建筑工程测量中的应用分析 [J].建材与装 饰,2018,(39):243-244.

[3] 何玲.测绘技术在现代工程测量中的应用探析 [J]. 山西建 筑,2019,45(08):186-187.

[4]彭安安.工程测量中 GIS技术数字化测绘技术的应用 [J].江西建 材,2019,(02):84-85.

\section{作者简介：}

王琅(1972--),男,福建省福州市人,汉族,本科,工程师,研究方向: 测绘。 